All patients underwent baseline magnetic resonance angiography (MRA) and a follow-up MRA at least one year after baseline per a standardized imaging protocol. The presence of angiographic lesions, defined as stenosis, occlusion, or aneurysm, was evaluated by visual inspection by a single reader who was blinded to clinical status. Angiographic lesions were evaluated in 4 segments of the aorta and in 13 branch arteries. On follow up angiography, the development of new lesions was recorded, and existing lesions were characterized as improved, worsened, or unchanged.

Results: 782 arterial territories were evaluated from 46 patients with LVV (TAK=28; $\mathrm{GCA}=18$ ). Baseline characteristics were as follows: Age [TAK=24.8 years (18.6-34.9), GCA=64.8 years (57.8-73.9)], Female gender [TAK=21 patients $(78 \%), G C A=16$ patients $(84 \%)$ ], Disease duration [TAK $=2.3$ years $(0.6-4.9)$, GCA 1.2 years (0.4-2.9)], Active clinical disease [TAK=12 patients (44\%), GCA 12 patients $(63 \%)$ ]. The median time from initial MRA to follow up MRA was 2.4 years (1.5-3.1) for GCA and 1.6 years (1.3-3.3) for TAK.

There were 159 territories affected at the baseline visit in 41 patients [TAK: 108 territories in 26 patients, GCA: 51 territories in 15 patients]. The development of new territory involvement was infrequent and only occurred in patients with TAK (8 new lesions out of 352 baseline unaffected territories (2.3\%) in 5 patients). At follow up, existing arterial lesions improved in 25 (15.7\%) territories, worsened in $6(3.8 \%)$ territories, and stayed the same in $128(80.5 \%)$ territories. There were no significant differences in angiographic progression of disease between the two diseases: improved - TAK 19 (17.6\%), GCA 6 (11.8\%); worsened - TAK 5 (4.6\%), GCA 1 (1.9\%); unchanged - TAK 84 (77.8\%), GCA 44 (86.3\%). Change in the branch arteries was more dynamic than change in the aorta (Figure). Improvement in angiographic disease was observed in $8(17 \%)$ patients (TAK=6, $\mathrm{GCA}=2)$. Worsening of disease was seen in $3(7 \%)$ patients $(\mathrm{TAK}=2, \mathrm{GCA}=1)$. In $5(11 \%)$ patients $(\mathrm{TAK}=5, \mathrm{GCA}=0)$, there were areas of improvement and other areas of worsening disease within the same patient.

Conclusion: Dynamic change in arterial lesions is observed in patients with TAK and GCA. Improvement and worsening of arterial lesions can be observed over time, even within the same patient. This observation suggests that both local factors at the level of the artery and systemic factors (e.g. treatment response) are likely associated with angiographic progression. The development of new angiographic lesions was infrequent, and only occurred in patients with TAK. These data may inform future guideline recommendations for angiographic monitoring in LVV. References: N/A

Figure.

Change in Arterial Disease Over Time in Large-Vessel Vasculitis

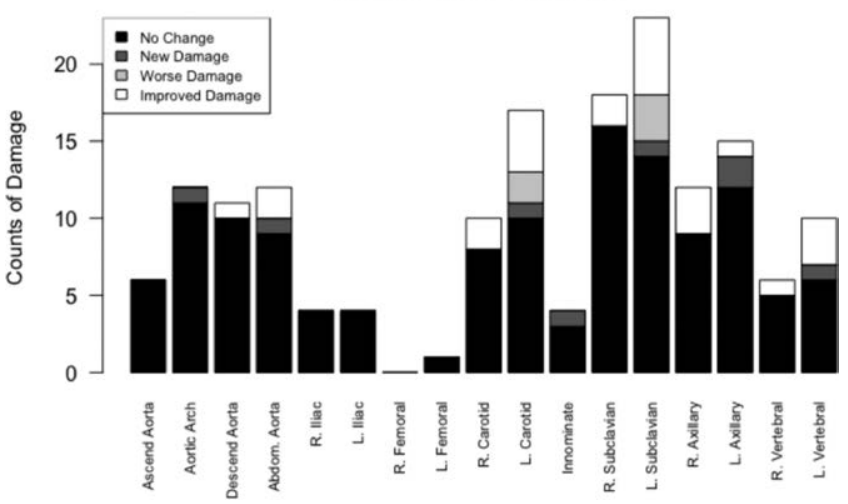

Disclosure of Interests: None declared DOI: 10.1136/annrheumdis-2020-eular.1569

\section{SAT0252 INCREASED MORTALITY FOR INDIVIDUALS WITH GIANT CELL ARTERITIS: A POPULATION-BASED STUDY}

L. Barra ${ }^{1}$, J. Pope ${ }^{1}$, P. Pequeno ${ }^{2}$, J. Gatley ${ }^{2}$, J. Widdifield ${ }^{3} .{ }^{1}$ Western University, London, Canada; ${ }^{2}$ ICES, Toronto, Canada; ${ }^{3}$ Sunnybrook Research Institute, Toronto, Canada

Background: Individuals with giant cell arteritis (GCA) are at increased risk of serious morbidity including cardiovascular disease and stroke. Yet the risk of mortality among individuals with GCA have produced conflicting reports ${ }^{1}$.

Objectives: Our aim was to evaluate excess all-cause mortality among individuals with GCA relative to the general population over time.

Methods: We performed a population-based study in Ontario, Canada, using health administrative data among all individuals 50 years and older. Individuals with GCA were identified using a validated case definition (81\% PPV, 100\% specificity). All Ontario residents aged 50 and above who do not have GCA served as the General Population comparators. Deaths occurring in each cohort each year were ascertained from vital statistics. Annual crude and age/sex standardized all-cause mortality rates were determined for individuals with and without GCA between 2000 and 2018. Standardized mortality ratios (SMRs) were calculated to measure relative excess mortality over time. Differences in mortality between sexes and ages were also evaluated.

Results: Population denominators among individuals 50 years and older with GCA and the General Population increased over time with 12,792 GCA patients and $5,456,966$ comparators by 2018 . Annual standardized mortality rates among the comparators steadily declined over time and were significantly lower than GCA morality rates (Figure). Annual GCA mortality rates fluctuated between 42-61 deaths per 1000 population (with overlapping confidence intervals) during the same time period. SMRs for GCA ranged from $1.28(95 \% \mathrm{Cl} 1.08,1.47)$ at the lowest in 2002 to $1.96(95 \% \mathrm{Cl} 1.84,2.07)$ at the highest in 2018. GCA mortality rates and SMRs were highest among males and younger age groups.

Conclusion: Over a 19-year period, mortality has remained increased among GCA patients relative to the general population. GCA mortality rates were higher among males and more premature deaths were occurring at younger age groups. In our study, improvements to the relative excess mortality for GCA patients over time (mortality gap) did not occur. Understanding cause-specific mortality and other factors are necessary to inform contributors to premature mortality among GCA patients. References:

[1] Hill CL, et al. Risk of mortality in patients with giant cell arteritis: a systematic review and meta-analysis. Semin Arthritis Rheum. 2017;46(4):513-9.

Figure.

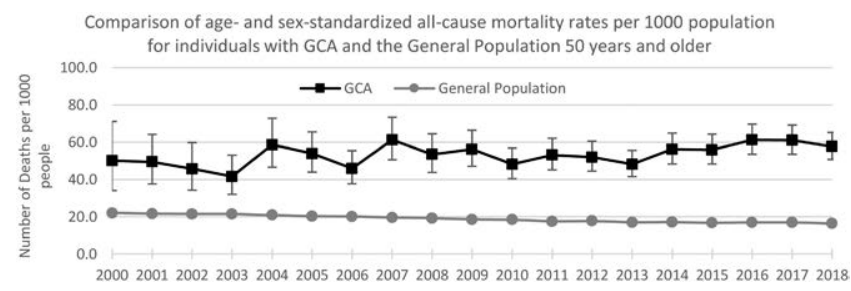

Acknowledgments: : This study was supported by a CIORA grant

Disclosure of Interests: Lillian Barra: None declared, Janet Pope Grant/ research support from: AbbVie, Bristol-Myers Squibb, Eli Lilly \& Company, Merck, Roche, Seattle Genetics, UCB, Consultant of: AbbVie, Actelion, Amgen, Bayer, Boehringer Ingelheim, Bristol-Myers Squibb, Eicos Sciences, Eli Lilly \& Company, Emerald, Gilead Sciences, Inc., Janssen, Merck, Novartis, Pfizer, Roche, Sandoz, Sanofi, UCB, Speakers bureau: UCB, Priscila Pequeno: None declared Jodi Gatley: None declared, Jessica Widdifield: None declared DOI: 10.1136/annrheumdis-2020-eular.1879

\section{SAT0253 \\ ANTI-NEUTROPHIL CYTOPLASMATIC ANTIBODIES PREDATE SYMPTOM ONSET OF ANCA-ASSOCIATED} VASCULITIS. A CASE-CONTROL STUDY

E. Berglin ${ }^{1}$, A. J. Mohammad ${ }^{2,3}$, J. Dahlqvist ${ }^{4}$, C. Eriksson ${ }^{5}$, J. Sjöwall ${ }^{6}$ S. Rantapää Dahlqvist ${ }^{5} .{ }^{1}$ Umeå University, Deps of Public Health and Medicine/ Rheumatology, Umeå, Sweden; ${ }^{2}$ University of Cambridge, Cambridge, United Kingdom; ${ }^{3}$ Lund University, Lund, Sweden; ${ }^{4}$ Uppsala University, Uppsala, Sweden; ${ }^{5}$ Umeå University, Umeå, Sweden; ${ }^{6}$ Linköping University, Linköping, Sweden

Background: Presence of anti-neutrophil cytoplasmatic autoantibodies (ANCA) is important for the diagnosis of ANCA-associated vasculitis (AAV) and reflects on-going immune processes. The timing of the antibody development and its contribution to disease is not well established.

Objectives: To investigate the presence of proteinase 3 (PR3)- and myeloperoxidase (MPO)-ANCA in blood samples collected from healthy individuals who subsequently developed AAV.

Methods: The Swedish National Patient Register of inpatient care and the Swedish Cause of Death Register were used to identify individuals assigned ICD codes for AAV (1) in the discharge summary or cause of death, respectively. The resulted cohort was then linked to the registers of 4 different biobanks to identify those with available predating blood samples. Diagnoses of AAV were confirmed and time point for onset of symptoms was identified by reviewing all available case records (1); 68 were classified as granulomatosis with polyangiitis (GPA), 14 as microscopic polyangiitis (MPA), and 4 as eosinophilic GPA (EGPA). The 86 cases (36 males, 50 females) had a mean (SD) age of 51.9 (16.9) years at sampling, with $\geq 1$ sample ( $26 \%$ plasma, $74 \%$ serum samples). The sampling time point before onset of symptoms was mean (SD); 4.4 (3.1) years. Serum and plasma control samples ( $n=198 ; 82$ males, 116 females; mean age (SD); 52.0 (16.5) years) were identified and matched for sex, age and date of sampling. The samples were first screened for ANCA using high sensitive ELISA (ORGANTEC diagnostika, Germany) and samples close to or above cut-off level were further analysed for capture PR3- and capture MPO-ANCA (ELISA; SVAR Life Science 
Sweden). For each case one control sample was included for the ANCA specificity tests. Statistical calculations were performed using SPSS software.

Results: In ANCA-screen $36.0 \%$ of the cases and $2.6 \%$ of controls tested positive ( $p<0.001$ ). $23 / 52(44.2 \%$ ) of the cases were PR3-ANCA positive (OR 56.3; $95 \% \mathrm{Cl} 7.26-436.62)$ and 8/52 (15.4\%) were MPO-ANCA positive (OR 4.18; $95 \%$ Cl 1.05-16.62). The mean (SD) predating time for PR3-ANCA positivity was 3.73 (3.49) years and for MPO-ANCA positivity 2.11 (1.46) years. Cases with positive predating PR3-ANCA were younger $(46.0 \pm 19.4$ vs $65.6 \pm 12.0$ years; $P<0.001)$ than cases with a negative predating PR3-ANCA. MPO-ANCA positive vs. MPOANCA negative pre-dating cases had more often severe disease (kidney/lung/ peripheral nervous system) (OR 15.08; 95\% Cl 1.68-135.54) at disease onset. Furthermore, predating MPO-ANCA positive vs predating PR3-ANCA positive cases had significantly more often severe manifestations at disease onset $(87.5 \%$ vs $28.6 \%$; $p$ 0.05). Cases positive vs. negative for MPO-ANCA in predating samples were less often classified as GPA ( $37.5 \%$ vs $86.4 \%$; $p<0.01)$ and more often as MPA (62.5\% vs $13.6 \%$; $p<0.05)$

Conclusion: The production of both PR3 and MPO-ANCA starts already years before onset of symptoms of AAV. Presence of MPO-ANCA appeared closer to symptom onset and with more severe disease presentation. Differences in the disease phenotype and disease severity were evident between the two ANCA serotypes.

References:

[1] Watts et al. Ann Rheum Dis 2007;66:222-22

Acknowledgments: : Vasculitis Foundation, USA

Disclosure of Interests: Ewa Berglin: None declared, Aladdin J Mohammad Speakers bureau: lecture fees from Roche and Elli Lilly Sweden, PI (GiACTA study), Johanna Dahlqvist: None declared, Catharina Eriksson: None declared, Johanna Sjöwall: None declared, Solbritt Rantapää Dahlqvist: None declared DOI: 10.1136/annrheumdis-2020-eular.2799

\section{SAT0254 \\ PROSPECTIVE ANALYSIS OF THE PREVALENCE OF GIANT CELL ARTERITIS IN CONSECUTIVE PATIENTS WITH POLYMYALGIA RHEUMATICA}

L. C. Burg ${ }^{1}$, C. Behning ${ }^{2}$, P. Brossart ${ }^{3}$, V. S. Schäfer ${ }^{1} .{ }^{1}$ Clinic for Internal Medicine III, University Hospital Bonn, Rheumatology, Bonn, Germany; ${ }^{2}$ University Hospital Bonn, Institute for Medical Biometry, Informatics and Epidemiology, Bonn, Germany; ${ }^{3}$ Clinic for Internal Medicine III, University Hospital Bonn, Oncology, Haematology and Rheumatoloy, Bonn, Germany

Background: Giant cell arteritis (GCA) is the most common form of systemic vasculitis affecting people aged 50 years and older. ${ }^{1}$ Although it is known, that GCA often coexists with polymyalgia rheumatica (PMR) ${ }^{2}$, prevalence of GCA in consecutive patients with PMR has not been investigated

Objectives: To prospectively examine the prevalence of GCA in consecutive patients with PMR by vascular ultrasound (US).

Methods: Patients with newly diagnosed PMR fulfilling the ACR /EULAR classification criteria $^{3}$ were included. Vascular US examination of the extracranial arteries typically involved in GCA, such as axillary arteries, vertebral arteries, common carotid arteries, superficial temporal arteries with both frontal and parietal branches, occipital arteries, facial arteries and the central retinal arteries was performed in all PMR patients. Diagnosis of GCA was made, if intima-media thickness (IMT) was above respective cut-off values. ${ }^{4}$

Results: Fifty patients with diagnosis of PMR underwent vascular US. Twenty-three patients (46\%) had PMR without GCA (PMR-group). The mean age in this group was 71 years $(S D \pm 10)$ with seventeen $(73 \%)$ females. In twenty-seven PMR patients (54\%) GCA was diagnosed (GCA-PMR group); the mean age in this group was 74 years $(S D \pm 9)$ with ten $(37 \%)$ females respectively. Mean C-reactive protein (CRP) values were $29.4 \mathrm{mg} / \mathrm{l}(\mathrm{SD} \pm 24.5)$ in the PMRgroup and $52.2 \mathrm{mg} / \mathrm{l}(\mathrm{SD} \pm 43.2)$ in the GCA-PMR-group. Although different mean values between the PMR-group and the GCA-PMR-group were observed, CRP values did not differ significantly between the two groups $(p=0.1432)$. Ten $(37 \%)$ patients of the GCA-PMR group did not have GCA symptoms and diagnosis of GCA was only determined by ultrasound examination. Symptoms and numbers of patients with respective symptoms are depicted in Table 1 and 2.

Table 1. Symptoms and signs in both groups

\begin{tabular}{lcc}
\hline Symptoms and signs & Group & \\
\hline & PMR-group & GCA-PMR-group \\
\hline Morning stiffness & $22(95 \%)$ & $23(85 \%)$ \\
$\geq 1$ shoulder with synovits or bursitis trochanterica & $12(52 \%)$ & $13(48 \%)$ \\
$\geq 1$ shoulder or hip with synovitis or bursitis & $11(48 \%)$ & $14(51 \%)$ \\
hip pain & $23(100 \%)$ & $23(85 \%)$ \\
No other joints affected & $22(95 \%)$ & $26(96 \%)$ \\
\hline
\end{tabular}

PMR-group: patients with diagnosis of polymyalgia rheumatica only

GCA-PMR-group: patients with diagnosis of polymyalgia rheumatic and giant cell arteritis
Conclusion: Prevalence of GCA in patients with PMR in our cohort was 54\%. Ten (37\%) patients with GCA and PMR did not have any GCA symptoms. Performing vascular US in patients with PMR can be useful to diagnose a clinical inapparent GCA. Prompt onset of the respective therapy could prevent complications of GCA and improve disease outcome.

References:

[1] Warrington KJ, Matteson EL. Management guidelines and outcome meas ures in giant cell arteritis (GCA). Clin Exp Rheumatol 2007;25:137-41

[2] Salvarani C, Cantini F, Hunder GG. Polymyalgia rheumatica and giant-cell arteritis. The Lancet 2008;372:234-45.

[3] Dasgupta B, Cimmino MA, Kremers HM, et al. 2012 Provisional classification criteria for polymyalgia rheumatica: a European League Against Rheumatism/American College of Rheumatology collaborative initiative. Arthritis Rheum 2012;64:943-54.

[4] Schäfer VS, Juche A, Ramiro S, Krause A, Schmidt WA. Ultrasound cut-off values for intima-media thickness of temporal, facial and axillary arteries in giant cell arteritis. Rheumatology (Oxford) 2017;56:1479-83.

Table 2. Number of patients in each group with symptoms of giant cell arteritis

\begin{tabular}{lcc}
\hline Symptoms & Group & \\
\hline & PMR-group & GCA-PMR-group \\
\hline Visual symptoms & $2(9 \%)$ & $8(30 \%)$ \\
Headache & $2(9 \%)$ & $9(33 \%)$ \\
Jaw claudication & $4(18 \%)$ & $10(38 \%)$ \\
Scalp tenderness & $0(0 \%)$ & $5(19 \%)$ \\
No GCA symptoms & $15(65 \%)$ & $10(37 \%)$ \\
\hline
\end{tabular}

PMR-group: patients with diagnosis of polymyalgia rheumatica only

GCA-PMR-group: patients with diagnosis of polymyalgia rheumatic and giant cell arteritis

Disclosure of Interests: None declared

DOI: 10.1136/annrheumdis-2020-eular.5497

\section{SAT0255 THE RATE OF RELAPSE AMONG PATIENTS WITH LARGE VESSEL VASCULITIS AND THE SYSTEMIC INFLAMMATORY RESPONSE AS A POSSIBLE PREDICTOR FOR RELAPSE}

V. M. Coroian ${ }^{1}$, S. Saur ${ }^{1}$, A. C. Pecher ${ }^{1}$, T. Xenitidis ${ }^{1}$, J. Henes ${ }^{1} .{ }^{1}$ Centre for Interdisciplinary Clinical Immunology, Rheumatology and Auto-inflammatory Diseases (INDIRA) and Department of Internal Medicine II (Oncology, Haematology, Immunology and Rheumatology), University Hospital Tuebingen, Germany, Tübingen, Germany

Background: Large vessel vasculitides are known relapsing diseases. However the rate of relapses has been seldom addressed and there are only few data on relapse predictors.

Objectives: We conducted the present study to investigate the prevalence of relapses in the first year after diagnosis and the overall relapse among patients with large vessel vasculitis. Furthermore, we aimed to identify if the systemic inflammatory response (SIR) is a possible predictor for relapse among patients with large cell vasculitis

Methods: The systemic inflammatory response (SIR) has been described as a potential clinical and serological score predicting the risk for relapses ${ }^{1}$. SIR estimates the systemic inflammatory activity at the time point of first diagnosis ${ }^{1}$. It was defined as follows: Temperature $>38^{\circ} \mathrm{C}$, weight loss $>4 \mathrm{~kg}$, Haemoglobin $<11 \mathrm{~g} / \mathrm{dl}$ and erythrocyte sedimentation rate $>85 \mathrm{~mm} / \mathrm{h}$. For each of the above-mentioned criteria one point was attributed, leading to a range from 0 to 4 points. Patients with 3 to 4 points were considered having a highly inflammatory response and patients with an SIR $\leq 2$ were considered having a low inflammatory response and thus a lower risk for relapses. Relapses are defined as reappearance of disease-related symptoms requiring treatment adjustment. The study cohort included 75 patients with large cell vasculitis (Giant Cell Vasculitis Takayasu Vasculitis, inflammatory non-infections Aortitis), longitudinally followed by the authors over a mean period of $5.2 \pm 3.3$ years (range 1-14 yr).

Results: The study-cohort includes 71 patients with a mean age at diagnosis of $63.5(16-85)$ years. Almost three quarters $(73 \%)$ of the patients were women. Most of the patients were suffering from GCA (73.2\%), followed by Takayasu arteritis (16.9\%) and inflammatory non-infections Aortitis (9.8\%). 38 patients $(53.5 \%)$ relapsed at least once during the follow up, and 17 patients had two or more relapses. The vast majority of relapses $(86.8 \%)$ were observed within the first year following diagnosis. Most of the patients, 54 patients $(76 \%)$, were considered having a low inflammatory response (SIR $<=2$ ). The relapse rate in this group was $59.2 \%$. On the other hand, there were 17 patients having an SIR higher or equal to 3 points. The relapse rate in this group was $33 \%$.

Conclusion: In conclusion, the results of this preliminary study reveal that the relapse rate among patients with large vessel vasculitis high is. The SRI appears to be an inadequate predictor for relapse in this cohort. 